\title{
Generating Classes of 3D Virtual Mandibles for AR-Based Medical Simulation
}

Neha R. Hippalgaonkar; Alexa D. Sider;

Felix G. Hamza-Lup, PhD; Anand P. Santhanam, PhD; Bala Jaganathan, MD, MHA; Celina Imielinska, PhD; Jannick P. Rolland, PhD

\begin{abstract}
Introduction: Simulation and modeling represent promising tools for several application domains from engineering to forensic science and medicine. Advances in 3D imaging technology convey paradigms such as augmented reality (AR) and mixed reality inside promising simulation tools for the training industry.

Methods: Motivated by the requirement for superimposing anatomically correct 3D models on a human patient simulator (HPS) and visualizing them in an AR environment, the purpose of this research effort was to develop and validate a method for scaling a source human mandible to a target human mandible within a $2 \mathrm{~mm}$ root mean square (RMS) error.

Results: Results show that, given a distance between 2 same landmarks on 2 different mandibles, a relative scaling factor may be computed. Using this scaling factor, results show that a $3 \mathrm{D}$ virtual mandible model can be made morphometrically equivalent to a real target-specific mandible within a $1.30 \mathrm{~mm}$ RMS error.

Conclusion: The virtual mandible may be further used as a reference target for registering other anatomic models, such as the lungs, on the HPS. Such registration will be made possible by physical constraints among the mandible and the spinal column in the horizontal normal rest position.

(Sim Healthcare 3:103-1 10, 2008)
\end{abstract}

Key Words: Augmented reality, Morphometry.
M edical simulation techniques have become an important aid for understanding and perceiving the fundamentals of clinical procedures. Under the clinical envelope, key training applications such as the development of dynamic anatomy visualization for teaching ${ }^{1}$ and computer-assisted guidance in surgery ${ }^{2}$ couple medical simulation with visualization frameworks. The usage of subject-specific anatomy and physiology further enhances the medical simulation and visualization applications from training paradigm to diagnostics and subject-specific planning of clinical interventions. Examples of applications include visualization of human models under normal and diseased states and minimally invasive surgical guidance. ${ }^{3}$

The focus of our research is to integrate medical visualization techniques to the human patient simulator (HPS), a human-like plastic mannequin, which allows different respi-

From the Optical Diagnostics and Applications Laboratory (N.R.H., A.D.S.), the Department of Radiation Oncology (A.P.S.), the Institute of Simulation and Training (B.J.), the College of Optics and Photonics (A.P.S., J.P.R.), University of Central Florida, Orlando, FL; the School of Computing, Armstrong Atlantic State University, Savannah GA (F.G.H.-L.); M.D. Anderson Cancer Center, Orlando, FL (A.P.S.); and the Department of Bioinformatics, Columbia University, New York, NY (C.I.).

Supported by the NSF/ITR IIS-00-820-16, NSF/HCI 03-07189 the Link Foundation, the Office of Naval Research Grant N000140310677, the U.S. Army Simulation, Training, and Instrumentation Command (STRICOM), and the Florida Photonics Center of Excellence.

Reprints: Anand P. Santhanam, College of Optics and Photonics, University of Central Florida, 4000 Central Florida Blvd, Orlando, FL 32816. E-mail:

hellotoanand@gmail.com.

The authors have indicated that they have no conflicts of interest to disclose.

Copyright $(92008$ Society for Simulation in Healthcare

DOI: $10.1097 /$ SIH.0b013e31816b5d54 ration functionalities to be simulated. Specifically, the subject-specific 3D models are superimposed on a HPS and visualized in an augmented reality (AR) environment. In the context of generating an effective visualization of the airways of the HPS for example, the requirement to appropriately scale and register previously acquired 3D anatomy from a source human model to fit the HPS arises. The scaling of the mandible is a first step towards obtaining the 3D scaled internal anatomy for the HPS. In our approach, the mandible is used as a reference target for registering anatomic models, such as the lungs and the larynx, on the HPS. Such registration is made possible by physical constraints between the mandible and the spinal column in the horizontal normal rest position as further illustrated in the Discussion.

In this article, we discuss a method to scale a 3D mandible model to morphometrically fit the 3D mandible of the HPS. Once scaled, the 3D mandible model can be registered to the HPS chin using simple rigid registration techniques. ${ }^{4}$ This study demonstrates that, within a gender, race, and age group, it is feasible to scale two 3D high-resolution mandibles to make them morphometrically equivalent. Based on personal communications with medical experts, an overall morphometric equivalence within a $2 \mathrm{~mm}$ root mean square (RMS) error was clinically established to be sufficient for the development of human-scale AR training procedures. ${ }^{5}$ Such performance imposes an upper bound on the departure of 3D models from the internal anatomy of the HPS by construction. Given that the HPS follows the anatomy of a young adult male, in the selection of mandibles, we considered 3 male white subjects within the age group of 16- to 24-year- 
old where the mandible growth has attained its full maturity, and no degeneration has taken place. The methodology discussed in this article leads to the computation of a scaling factor for which 2 subject mandibles can be made morphometrically equivalent.

\section{RELATED WORK}

Morphometrics generally refers to the analysis of size and shape. ${ }^{6}$ Although size changes refer to a proportional increase or decrease in all dimensions of a 3D model, shape change refers to a change in the outline of the form under examination. ${ }^{7}$ There are 2 distinct groups of techniques in the current literature: landmark-based techniques and boundary outline techniques. Landmark-based techniques are based on establishing distance and angle relations between the landmarks. Boundary outline techniques on the other hand investigate the shape of the perimeter of a structure defined at a certain resolution.

Initial methods of landmark-based analysis were based on either a statistical or superimposition approach. In the case of a statistical approach, a suitable 3D model is chosen from a database of 3D models based on the landmark positions (eg, facial morphing ${ }^{8}$ ). In the case of a superimposition approach, the $3 \mathrm{D}$ models were scaled and rotated until the eigenvectors of the covariance matrix of landmarks of the two 3D models matched with preset tolerances. ${ }^{9}$ Using these methods, the local surface variations were not represented accurately. Deformation methods such as Finite Element Methods ${ }^{10}$ and Thin Plate Splines ${ }^{11}$ were used to estimate the shape difference between two 3D virtual models. The required deformation conveyed their morphologic differences. Surface reconstruction methods such as elliptical Fourier functions ${ }^{12}$ and medial axis methods were also used for boundary outline estimation analysis. These latter methods convey the shape changes between 2 given 3D virtual models effectively in terms of a set of spherical basis functions. The deformation and surface reconstruction methods provided measurements with high precision but lacked an established relationship to biology and statistical variations required for effective analysis. ${ }^{4,13}$

The biologic effect on the shapes of anatomic organs is better represented using Growth Allometry techniques, ${ }^{14}$ where the direction of growth of an anatomic structure based on race and age of a subject is taken into account. An approach to model the mandible bone growth along the surface of the mandible is presented in. ${ }^{14}$ Such methods were limited by the lack of mathematical flexibility and user-friendliness.

The most flexible and user-friendly methods for algebraic analysis are the Conventional Cephalometric Methods ${ }^{15}$ and analysis based on the landmarks linear distance, angles, and ratios. However these methods do not adequately represent the shape details and are not fully capable of evaluating shape and size. Euclidean distance matrix analysis (EDMA) was proposed as an improvement to these conventional methods. ${ }^{13}$ In this method biologic shapes are compared using landmark coordinate data by mathematically localizing their morphologic distances. The resultant is a set of ratios among the Euclidean distances.

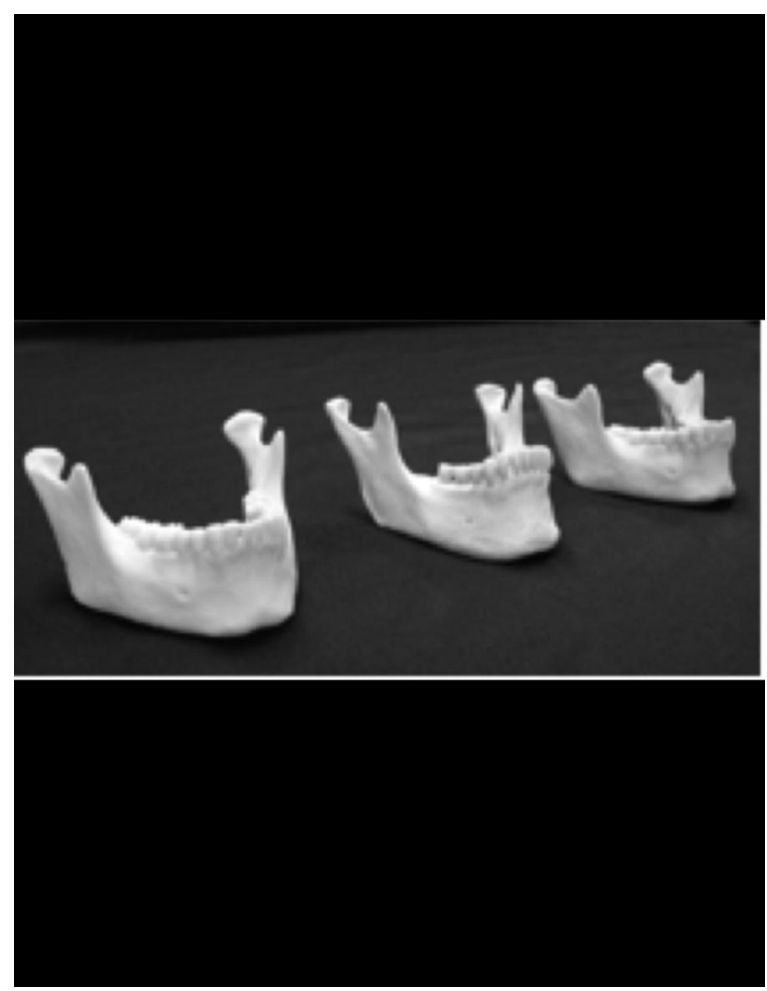

Figure 1. Adult white male mandibles.

\section{METHODS FOR VIRTUAL 3D MANDIBLE GENERATION}

The virtual 3D mandibles were generated via high resolution digitization (ie, a point was measured within $0.1 \mathrm{~mm}$ accuracy) From a set of digital 3D points, a 3D polygonal representation of the real object was obtained. The alternative would consist in segmenting CT models. In this first investigation we wanted to ensure the highest possible accuracy of the 3D models. ${ }^{16,17}$ When considering CT, one would want to quantify errors caused by both the limited resolution of the CT acquisition (ie, $\geq 2.5 \mathrm{~mm}$ ) and the segmentation process. Three adult white male mandibles shown in Figure 1 were obtained from Global-Technologies. ${ }^{18}$

The computer generated models corresponding to these mandibles were obtained through a nondestructive 2-step digitization process performed with an optical tracking system, the Optotrak $3020,{ }^{19}$ and its associated digitizing probe.

In the first step of digitalization, the position information from the mandible's surface was collected. Naturally, the resolution of the generated model was proportional to the number of points collected. For this particular experiment, we divided the mandible surface in 15 regions of different sizes, with larger sizes where the surface curvature was low. Regions slightly overlapped, and we collected about 2000 points from each region.

In the second step, the collected data was imported in the Geomagic Studio 5.0, ${ }^{20}$ a software package that allowed redundant point elimination and generated a $3 \mathrm{D}$ polygonal model. The points collected in the fifteen regions were merged for each mandible. The 3D polygonal models obtained consisting of $47 \mathrm{k}$ polygons each are shown in Figures $2 \mathrm{~A}$ to $2 \mathrm{C}$. 

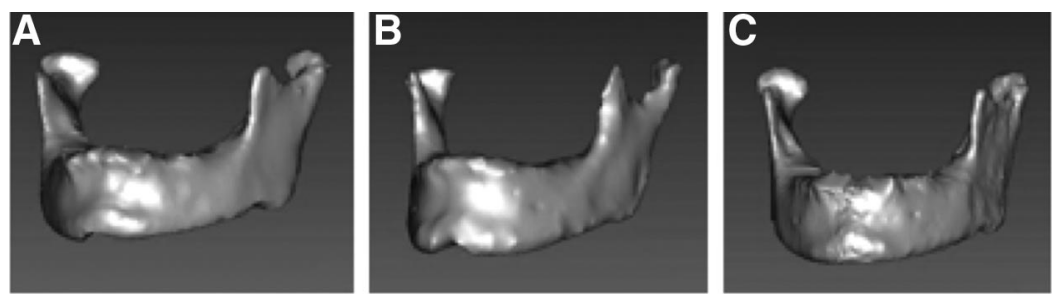

Figure 2. $3 \mathrm{D}$ virtual models of the three mandibles shown in Figure 1. (A) Mandible 1; (B) mandible 2 ; (C) mandible 3 .

\section{MANDIBLE SHAPE ANALYSIS}

We used a landmark-based shape analysis to scale a given mandible to a target mandible. Such a scaling approach satisfied the requirement of being able to put the models in coincidence within a $2 \mathrm{~mm}$ RMS error. Landmark selection, shape characterization, and scaling computation of one mandible to another are described in the following sections.

\section{Landmarks Selection}

There are 77 significant landmarks on the human mandible selected for clinical applications such as function regulator therapy, which deals with mandible repositioning and rotation. ${ }^{12,21,22}$ In this experiment 31 landmarks were chosen independently by one anatomist and one medical expert based on their biologic importance and the direction of growth. ${ }^{14,23}$ The 2 experts selected the same landmarks as a set with 100\% accordance. Specifically, they selected 3 landmarks (ie, number 1, 2, and 3) common to both the left and right sides of a real 3D mandible, and 14 additional landmarks per side, totaling 31 landmarks as shown in Figure 3. Note that the numbering of the landmarks is not the same as that provided in reference ${ }^{23}$; rather it follows a 1 to 31 numbering for the subset of selected landmarks.

The 2 experts then recorded the position of the 31 selected landmarks on each computer generated mandible in one session. Both experts repeated the procedure once for each mandible at least 2 weeks later, thus each landmark was identified with 2 independent markings per expert. In the localization of each landmark, the experts were asked to consider the spatial location of each landmark and to localize its centroid. To determine the most accurately chosen landmarks we imported all 4 sets of landmarks on top of the digitized mandible model and we determined for each landmark the average of its Cartesian coordinates as well as its standard deviation based on the available datasets (ie, 4 markings total per landmark) Among all pairs of landmarks, of interest was not only their accurate marking but also their palpability given that in the final application the landmarks need to be marked on a HPS. Among the easily palpable landmarks, landmarks 1 and 7 were found to be the most accurate, and thus, their distance was chosen as the reference length used for shape characterization.

Having repeated landmark selection within expert and 2 experts provides us with some measure of inter- and intraexpert variability among experts in the generation of a surrogate of ground truth for landmarks location on the mandible. ${ }^{24}$ In deciding on the minimum required number of experts needed for a given task, we showed in another application related to a more complex task, that in segmentation of 3D models, it was sufficient for 3 experts to repeat delineations 3 times to assess intra- and interexpert variability for ground truth generation. ${ }^{25}$ Hand marking by experts, conducted under the same strict protocol, is still the best method to generate ground truth for the landmarks on the mandible. The choice for 2 experts in the marking of landmarks is consistent with the task complexity. A more extensive follow-up study could focus on selecting and digitizing a larger number of mandibles to show variation of human anatomy in different phenotypes, as well as including more experts.

\section{Mandible Shape Characterization}

We tailored the EDMA method to the 3D mandible models generation. The distances between landmarks were computed along the surface of the 3D models using geodesic distances. ${ }^{26-28}$ The strength of this method lies in combining the mathematical simplicity of the EDMA method discussed in ${ }^{13}$ with the biologic growth statistics of the mandible. ${ }^{13}$ The work exemplifies translational research driven by a medical application where known methodologies in different areas combined together, as in our case: biomathematics, biology, imaging, modeling, 3D visualization/virtual reality and morphometry are tailored to serve best the application. Transla-

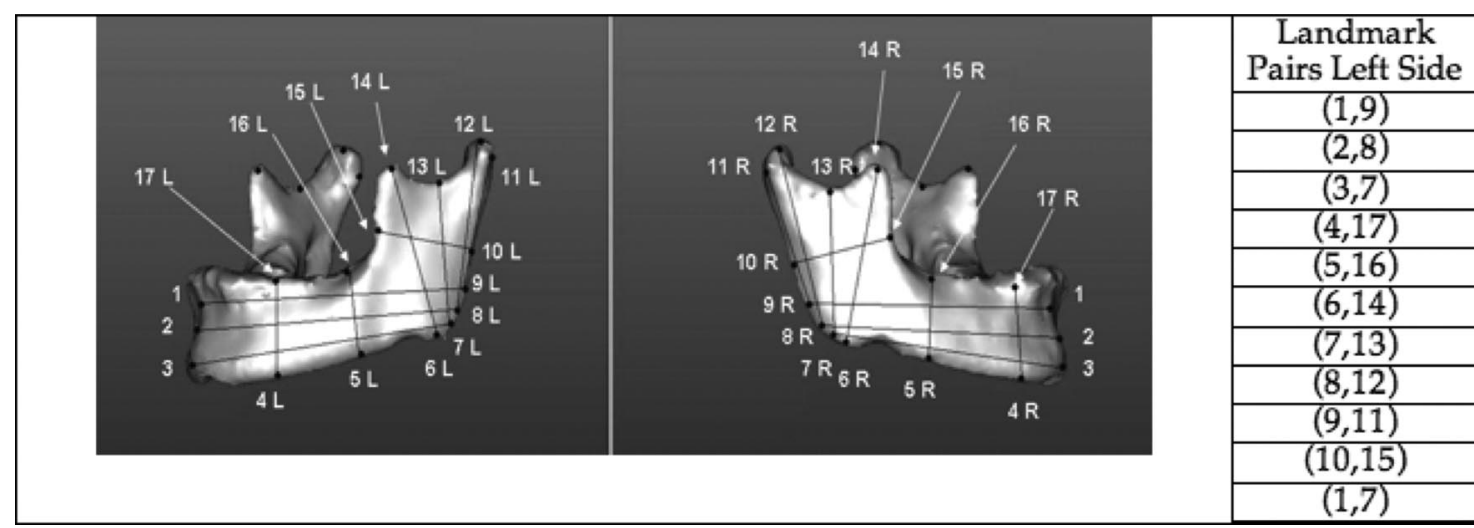

Figure 3. Landmark selection and landmark pairs. 


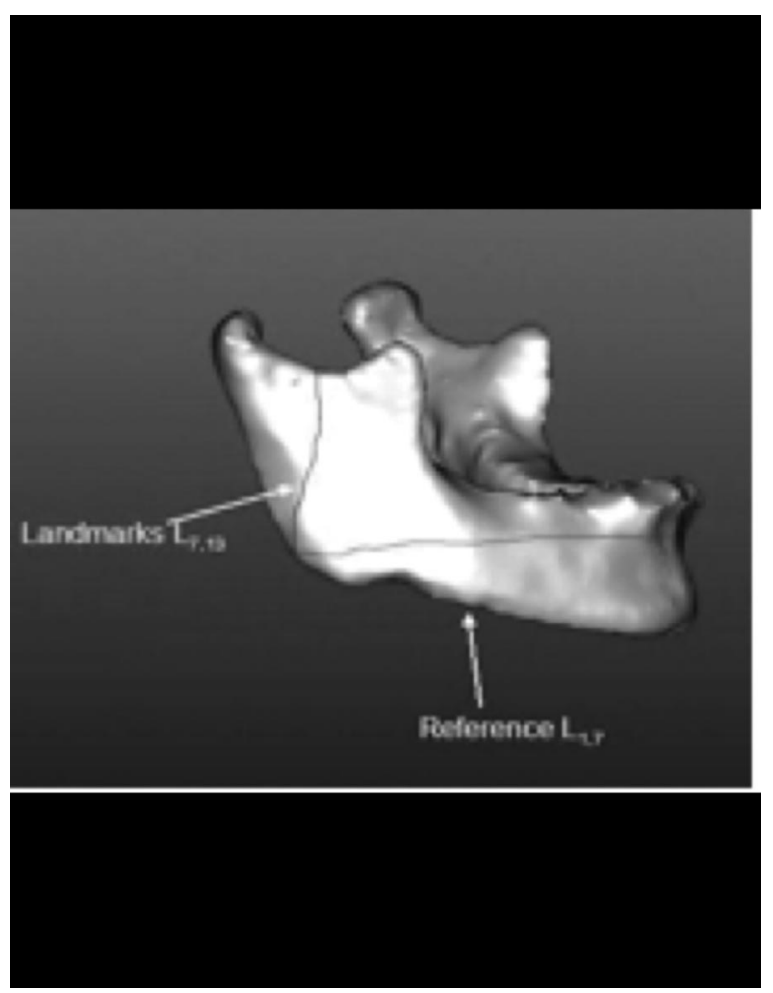

Figure 4. Local ratio.

tional research in conjunction with well stated medical challenges can serve 2 causes: solve a sound medical problem and generate potential new open problems in the component areas.

To proceed with the characterization of each mandible, landmark pairs based on the direction of growth of the mandibles were formed, ${ }^{14}$ yielding 11 pairs. The landmark pairs are shown in Figure 3.

We then define 2 types of ratios:

- The Local Ratios, denoted as $L R_{i, j}$, are the ratios of the length $L_{i, j}$ between selected pairs of landmarks $i$ and $j$ to the reference length $R$ on the same mandible as illustrated in Figure 4 (ie, $L R_{i, j}=L_{i, j} / R$ ).

- The Global Ratios, denoted as $G R_{i, j}$, are the ratios of the local ratios for any pair of landmarks across any 2 man-

\section{Expert 1}

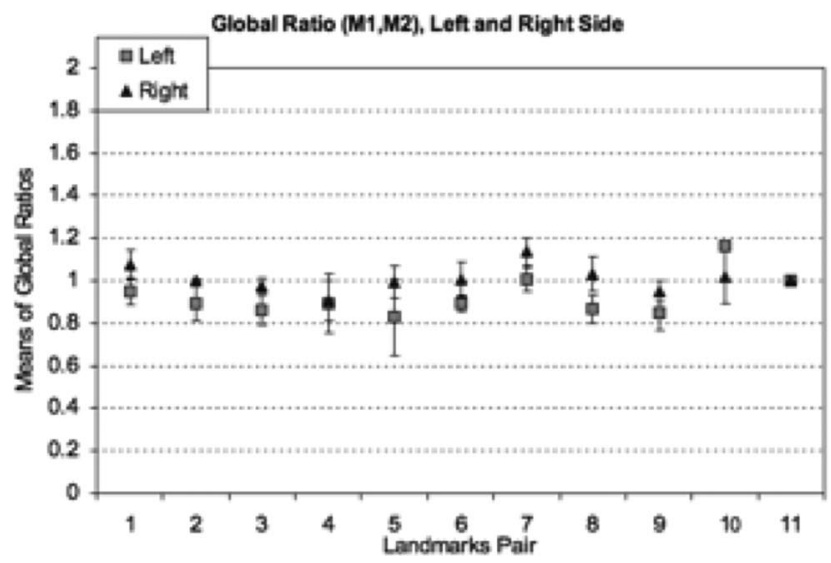

dibles. The hypothesis is that they will be approximately 1 indicating that the local ratios are equal. The $G R_{i, j}$, may be expressed as

$$
G R_{i, j}=\frac{L R_{i, j} \text { for mandible } a}{L R_{i, j} \text { for mandible } b}
$$

The geodesic distance between the landmarks of each landmark pairs was computed using the GeoMagic distance tool. Further we normalized the distance between the landmark pairs by the distance between landmarks 1 and 7 denoted as R. Given the local ratios for each mandible, the set of global ratios was determined for each pair of mandibles ie, mandible 1 and 2 (Fig. 5), mandible 2 and 3 (Fig. 6), mandible 3 and 1 (Fig. 7). The left and the right sides of each mandible were treated independently to capture potential asymmetries and are represented on each figure with a square and a triangle symbol, respectively. The measurements are plotted for each expert separately on the left and right side, respectively.

Results show that the global ratios are 1 within a $\pm 20 \%$ range, with a RMS variation of $3 \%$. Such results suggest that the distances are proportional which leads us to the following hypothesis: if the distance between 2 landmarks on the mandible is given, which characterizes the growth under a particular age group, the distance between other landmarks can be computed. Such a relationship allows us to generate scaled 3D mandible models from a given mandible model. The plots in Figure 5 also point to the symmetry of the right and left sides of the mandibles because the global ratios for the left and right sides follow a similar trend.

To quantify the landmark selection we computed the average values of the local ratios over the 3 mandibles for each pair of landmarks. Figure 8 presents the plots of these averages for each anatomist in each session. Results show that these values are extremely close to each other, thus pointing to the consistency of the landmark selection process.

\section{Scaling Computation}

The method of scaling a generic mandible to a target mandible proceeded as follows. We first marked the positions of the reference landmarks pairs in the generic and target man-

\section{Expert 2}

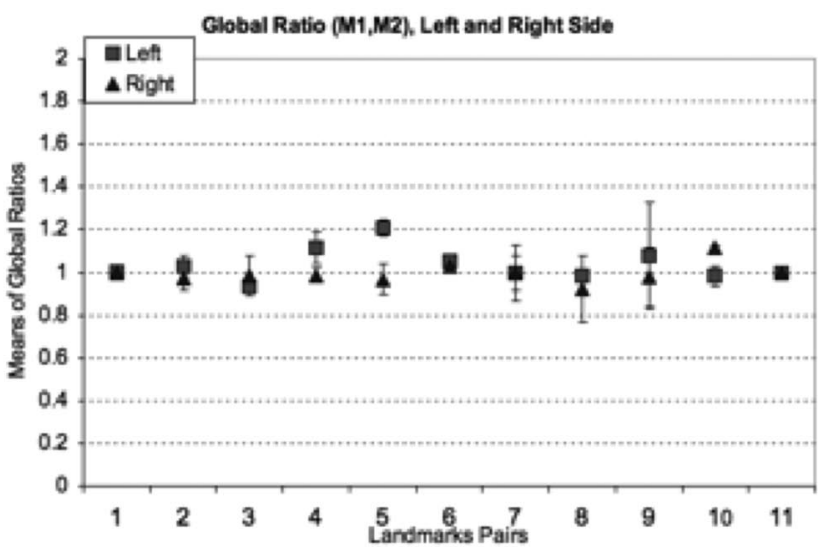

Figure 5. Global ratios for mandibles one and two, expert one on the left, expert two on the right. The landmark pairs are as labeled in Figure 3 . The global ratios of left and right sides of the mandibles are very close to each other and is in the proximity of one. 
Expert 1

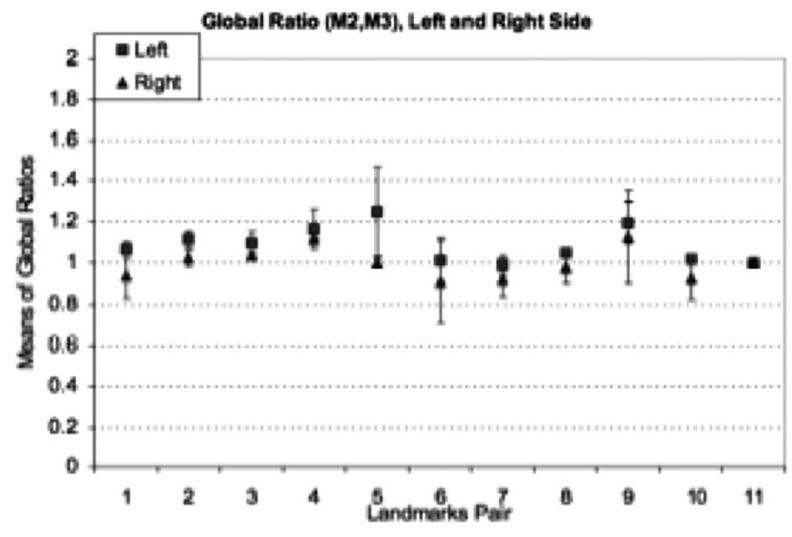

Expert 2

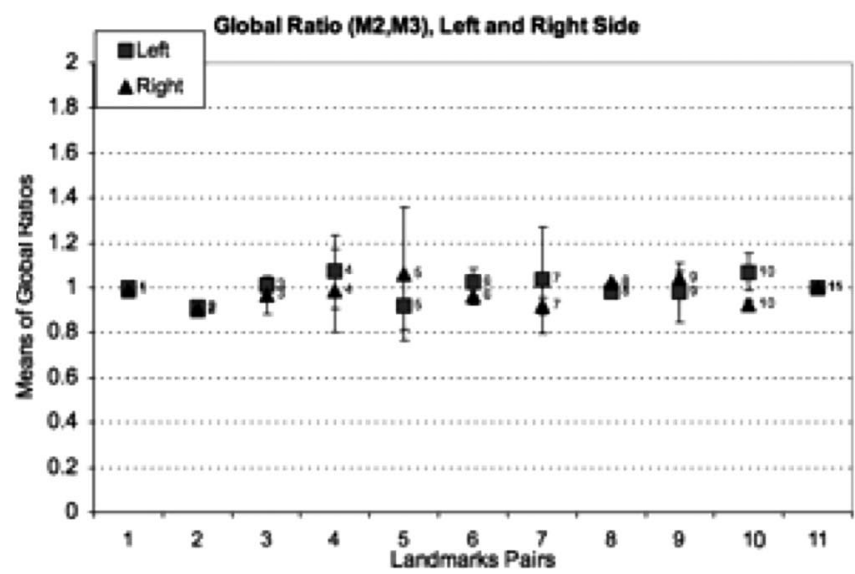

Figure 6. Global ratios for mandibles two and three, expert one on the left, expert two on the right. The landmark pairs are as labeled in Figure 3 . The global ratios of left and right sides of the mandibles are very close to each other and is in the proximity of one.

dibles and computed the distance between them along the surface of that mandible. We then computed the ratio of the reference landmarks pair lengths across the 2 mandibles. This ratio generated a relative scaling factor that was used to scale a given mandible to the target mandible on the directions of growth. As discussed in ${ }^{14}$, we established 2 main directions of growth with the point of dental-protruberance, taken as the origin. The directions are (i) vertical along the symphysis and (ii) horizontal along the midpoints of the 2 anterior masseter corners. Using this new coordinate system, the source mandible was scaled to the target mandible using the Geomagic software package.

\section{ANALYSIS OF VIRTUAL MODEL SIMILARITY}

To quantify the scaling process we compared a generated scaled virtual mandible model to real counterpart. For this experiment, we used 2 real mandibles denoted M1 and M2. The models had a level of similarity with an RMS departure of
$2.15 \mathrm{~mm}$ mean and $1.32 \mathrm{~mm}$ standard deviation. The quantification experiment consisted of the following steps:

1. Obtained a virtual model (VM1) for mandible M1 using the digitization process described earlier.

2. Scaled VM1 using the method proposed in Scaling Computation from M1 to M2. Let us call this generated virtual model VM1Scaled.

3. Obtained a virtual model (VM2) for mandible M2 using the same digitization process. This step was necessary only for the purpose of quantifying the similarity between the 2 virtual models VM2 and VM1Scaled.

4. Registered the mandible VM2 with VM1Scaled using a rigid body transformation with Geomagic software. The chin landmarks $(1,2$, and 3$)$ of both the mandibles were first associated. The VM1Scaled was then rotated along the vertical chin axis until the distances between the landmarks 7L and 7F of both mandibles were minimized.

5. Compared VM2 and VM1Scaled using an RMS error analysis between all the surface nodes of both mandibles.

\section{Expert 1}

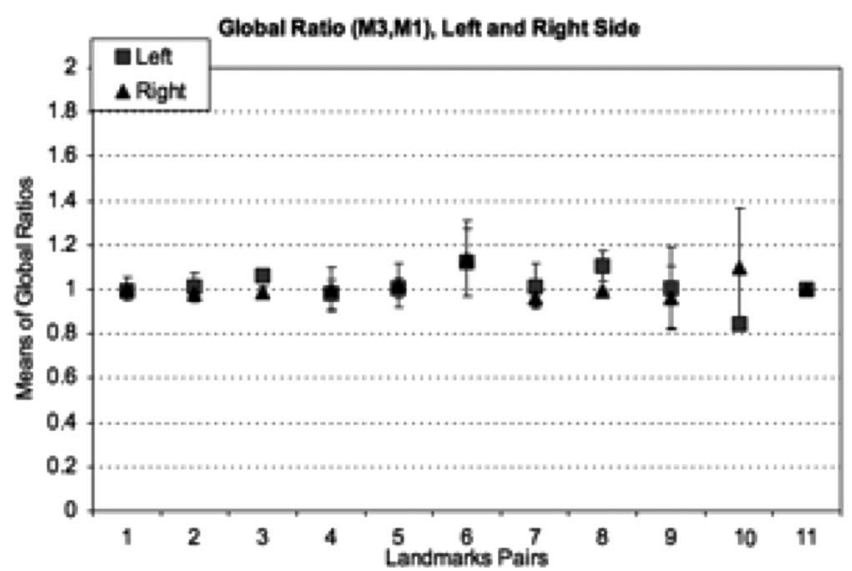

\section{Expert 2}

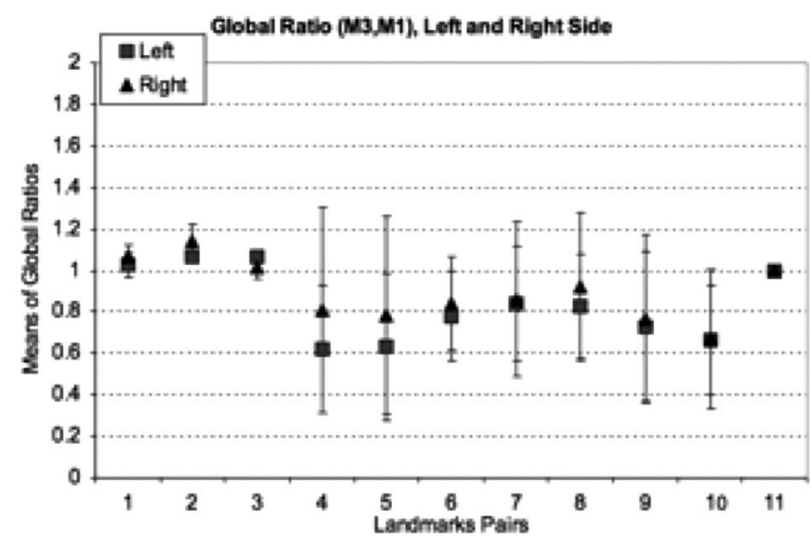

Figure 7. Global ratios for mandibles three and one, expert one on the left, expert two on the right. The landmark pairs are as labeled in Figure 3. The global ratios of left and right sides of the mandibles are very close to each other and is in the proximity of one. 


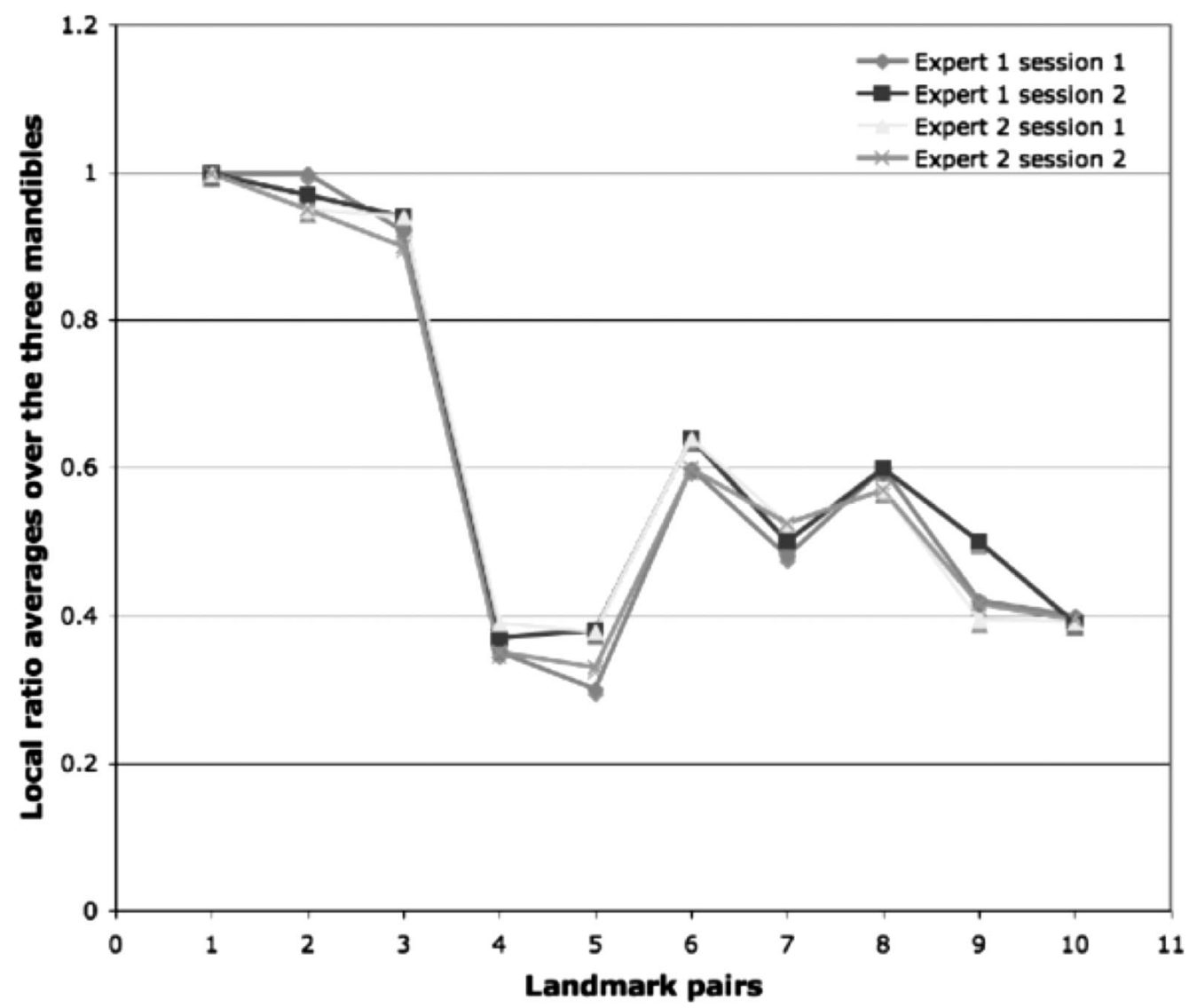

Figure 8. Local ratio averages over the three mandibles for each expert and session.

To perform a preliminary subjective visual assessment, one model was set of blue color and the other of gray color. Figure 9 provides a subjective assessment of the high similarity of the two $3 \mathrm{D}$ virtual models.

For a quantitative and objective assessment, once the reference landmarks were placed in coincidence, we computed the distance between each pair of vertices in the 3D models and drew a color distance-map that shows the areas of perfect fit (ie, light color) as well as the largest displacement (ie, dark color) between the vertices of the 2 models (Fig. 10).

As we can see in Figure 10, the light color is predominant in the images, which shows at these locations a perfect fit
Figure 9. VMIScaled in blue color superimposed over VM2 in gray color.

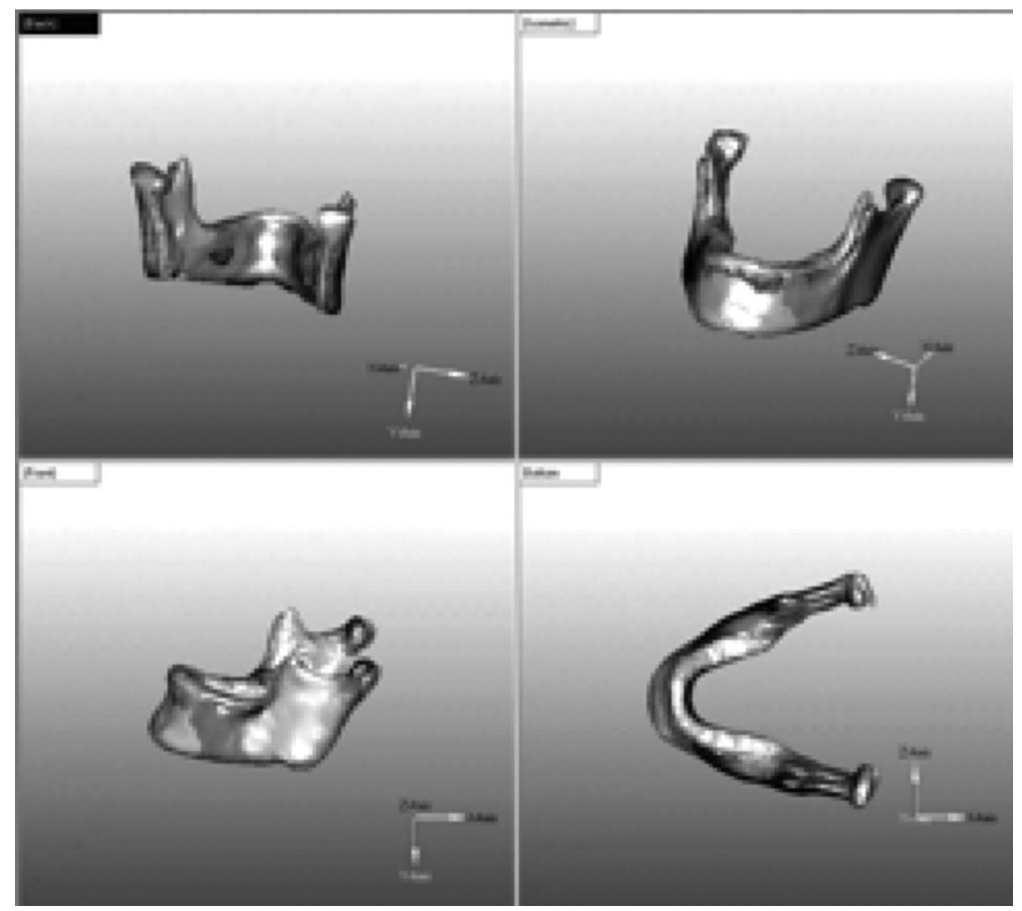




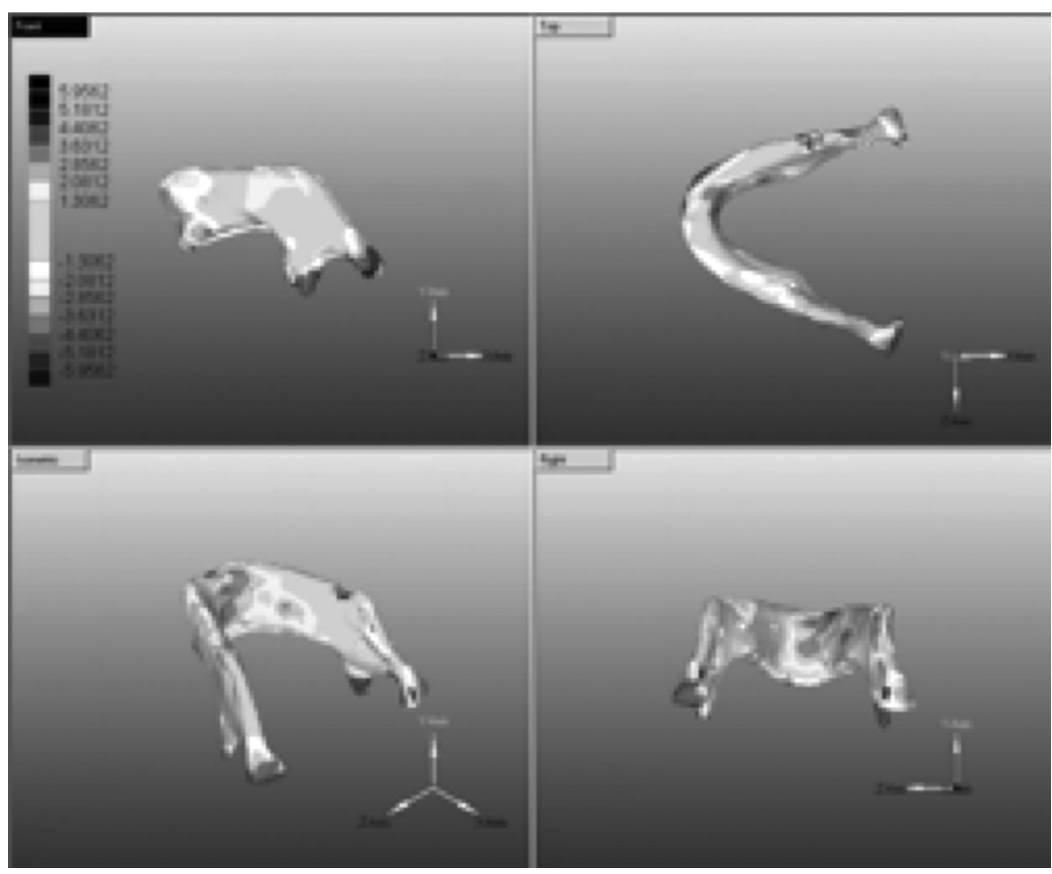

Figure 10. (VM1Scaled, VM2) Vertices distance map. between the 2 virtual models. Table 1 provides a quantitative summary of the results. It is important to note that in the experimental protocol, the fact that a similar digitization process was used in the quantification algorithm allowed us to ignore the digitization error terms because these errors compensated each other when the distance between the pair of vertices was computed.

Results show a level of similarity with an average departure of $1.30 \mathrm{~mm}$ within a $0.99 \mathrm{~mm}$ standard deviation. Such performance well satisfies the requirement of being able to put the models in coincidence within a $2 \mathrm{~mm}$ RMS error.

\section{DISCUSSION}

In this article we have presented a method and study on scaling a 3D mandible model to morphometrically fit the $3 \mathrm{D}$ mandible of the HPS. The method was validated with a source and target mandibles in the same age range ${ }^{16-24}$, gender and race. This scaling step forms the first step towards scaling the upper and lower airways of the HPS, which could significantly enhance training on the HPS.

We discussed a landmark-based shape analysis to scale a given mandible to a target mandible. Such a scaling approach satisfied the requirement of being able to put the models in coincidence within a 2-mm RMS error. We tailored the EDMA method to the 3D mandible models generation. The strength of this method is that it combines the mathematical simplicity of the EDMA method discussed in ${ }^{13}$ with the biologic growth statistics of the mandible discussed in. ${ }^{14}$

\section{Table 1. Comparison Statistics}

Number of polygons per model
Results discussed in this article constitute a preliminary proof that the distances are proportional, which leads us to the following hypothesis: if the distance between 2 landmarks on the mandible is given, which characterizes the growth under a particular race age and sex group, the distance between other landmarks can be computed. Such a relationship allows us to generate scaled 3D mandible models from a given mandible model.

The sample datasets used in this article correspond to white men at the age group 16 to 24 , where growth degenerations are not seen. Future work would involve the usage of mandibles of different age race and sex category. The usage of biologic growth statistics that corresponds to the mandibles of different age race and sex will also be investigated.

The work exemplifies translational research driven by a medical application where known methodologies in different areas combined together. Such translational research can serve 2 causes: solve a sound medical problem and generate potential new open problems in the component areas.

\section{CONCLUSION AND FUTURE WORK}

This article presents an alternative method to patient specific data acquisition of generating 3D models for augmented reality medical applications. We show that $3 \mathrm{D}$ anatomically correct mandible models can be scaled considering particular landmarks to be made morphometrically equivalent to each other. The method used to compute a scaling factor is simple in implementation, yet it could generate 3D mandible virtual models with vertices within a predicted $1.30 \mathrm{~mm}$ average error bound from their real counterparts. In the future we could investigate a larger number of mandibles that will represent different phenotypes categorized by gender, age, race and other to expand on the results found in this study. 


\section{ACKNOWLEDGMENTS}

The authors thank METI Corporation for providing through the U.S. Army with the upper torso of the Human patient simulator and Karen Kerner for the intubation position images using external anatomic landmarks.

\section{REFERENCES}

1. Argotti Y, Davis L, Outters V, et al. Dynamic superimposition of synthetic objects on rigid and simple-deformable objects. Comput Graph 2002;26:919-930.

2. Imielinska CP. Molholt. Incorporating $3 \mathrm{D}$ virtual anatomy into the medical curriculum. CACM 2005: 49-54.

3. State A, Keller K, Rosenthal M, et al. Stereo imagery from the UNC augmented reality system for breast biopsy guidance. Westwood J., ed. In: Medicine Meets Virtual Reality. CA: Newport Beach; 2003.

4. Sameshima GT, Melnick M, Singer J. Assessing ethnic differences in creaniofacial growth in response to treatment of malocclusion: a finite element approach. J Craniofac Genet Dev Biol 1997;17:48-56.

5. Discussion with medical experts. Department of Bio-informatics, C.U. New York, NY, 2003.

6. Lele S, Richtmeiser JT. Statistical models of morphometrics: are they realistic? Syst Zool 1990;39:60-69.

7. Kendall DG. A survey of the statistical theory of shape. Stat Sci 1989;4: $87-120$.

8. Romdhani S, Vetter T. Efficient, robust and accurate fitting of a 3D morphable model. In: Proceedings of the IEEE International Conference on Computer Vision, 2003.

9. Auffray JC, Debat V, Alibert P. Shape Asymmetry and developmental stability. In Chaplain MAJ, Singh GD, eds. On Growth and Form Spatio-Temporal Pattern Formation. Chichester: Wiley;1999:309-324.

10. Lozanoff S. Spenoethmoidal growth, malgrowth and midfacial profile. In Chapmain MA, Singh GD, eds. On Growth and Form Spatiotemporal Pattern Formation in Biology. Chichester: Wiley;1999:357372.

11. Bookstein FL. On the cephalometrics of skeletal changes. Am J Orthod 1982;15:305-314.

12. Chen SY, Lestrel PE, John W, et al. Describing shape changes in the human mandible using elliptical Fourier functions. Eur J Orthod 2000; 22:205-216.
13. Lele S, Richtmeiser JT. Euclidean distance matrix analysis: a coordinate-free approach for comparing biological shapes using landmark data. Am J Phys Anthropol 1991;9:77-91.

14. Bro-Nielsen M, Gramkow C, Kreiborg S. Non-rigid image registration using bone growth Model. In: CVRMed/ MRCAS'97. New York: Springer; 1997. Lecture Notes in Computer Science, pp. 1-12.

15. Steiner CC. The use of cephalometrics as an aid to planning and accessing orthodontic treatment. Am J Orthod 1990;46:721-735.

16. Collins R. 3D Imaging and Scanning Systems and Equipment. 2002. Available at: http://www.sculptor.org/3D/Scanning/imaging.htm.

17. GeoMagic. 3D Scanners Report. 2004.

18. Global Technolgies. 3D Mandible Molds-Rapid Prototyping. 2004.

19. Northern Digital Inc. 6DOF Optical Tracking System-Optotrak 3020. 2002.

20. GeoMagic Studio. Geomagic Studio Product Sheet. 2003.

21. Rashedi A. Evaluation of Vertical and sagittal growth in the mandible with deep bite angles in comparison with ideal vertical growth and the assessment of the activator treatment. Stockholm: Karolinska Institute; 1995:51-57.

22. Lestrel PE, Kerr WJS. Quantification of function regulator therapy using elliptical fourier functions. Eur J Orthod 1993;15:481-491.

23. Takeshita S, Sasaki A, Tanne K, et al. Moss: the nature of human craniofacial growth studied with finite element analytical approach. Clin Orthod Res 2001;4:148.

24. Udupa JK, Leblanc VR, Schmidt H, et al. A methodology for evaluating image segmentation algorithms. SPIE Med Imaging 2002; 4684:226-277.

25. Imielinska C, Rosiene Y, Jin Y. Ground truth evaluation of ischemic stroke hybrid segmentation in a rat model of temporary middle cerebral artery. Comput Assist Radiol Surg 2005;1281:74-79.

26. Mao Z, Siebert JP, Cockshott WP, et al. A coordinate free method for the analysis of 3D facial change. In: Medical Imaging 2004: SPIE, pp. $14-19$.

27. Hippalgaonkar N, Sider A. SAMAR-Scalable Anatomical Models for Augmented Reality. Presented at the 55th International Science and Engineering Fair, 2003, Washington, DC.

28. Hippalgaonkar N, Sider A. SAMAR-Phase II. Scalable Anatomical Models for Augmented Reality. Presented at the 56th International Science and Engineering Fair, 2004, Washington, DC. 